\title{
Meta
}

Journal des traducteurs

Translators' Journal

\section{La contribution de la pragmatique lexiculturelle dans un dictionnaire du tourisme}

\section{Marina Aragon Cobo}

Volume 50, numéro 4, décembre 2005

Pour une traductologie proactive - Actes

For a Proactive Translatology - Proceedings

Por una traductología proactiva - Actas

URI : https://id.erudit.org/iderudit/019908ar

DOI : https://doi.org/10.7202/019908ar

Aller au sommaire du numéro

Éditeur(s)

Les Presses de l'Université de Montréal

ISSN

0026-0452 (imprimé)

1492-1421 (numérique)

Découvrir la revue

Citer cet article

Aragon Cobo, M. (2005). La contribution de la pragmatique lexiculturelle dans un dictionnaire du tourisme. Meta, 50(4). https://doi.org/10.7202/019908ar
Résumé de l'article

Dans l'élaboration d'un dictionnaire du tourisme bilingue français-espagnol/espagnol-français, la pragmatique lexiculturelle peut avoir une place intéressante. Cet article se propose de montrer sa richesse et son utilité, car même des langues voisines peuvent présenter des écarts culturels qu'un dictionnaire doit cerner et expliciter, pour devenir un outil de réflexion et non seulement un instrument de traduction. 


\title{
La contribution de la pragmatique lexiculturelle dans un dictionnaire du tourisme
}

\author{
MARINA ARAGON COBO \\ Université d'Alicante, Alicante, Espagne \\ marina.aragon@ua.es
}

\begin{abstract}
RÉSUMÉ
Dans l'élaboration d'un dictionnaire du tourisme bilingue français-espagnol/espagnol-français, la pragmatique lexiculturelle peut avoir une place intéressante. Cet article se propose de montrer sa richesse et son utilité, car même des langues voisines peuvent présenter des écarts culturels qu'un dictionnaire doit cerner et expliciter, pour devenir un outil de réflexion et non seulement un instrument de traduction.
\end{abstract}

\begin{abstract}
When writing a bilingual dictionary (French-Spanish/Spanish-French) on tourism, the pragmatic dimension of words cannot be disregarded. The purpose of this paper is to examine its potentialities, which can be very practical and useful when analysing stylistic deviations in the meaning of words coming from languages that share common cultural and historical origins. Modern dictionaries, if they aim at becoming useful tools for translators, cannot ignore the so-called lexico-pragmatic dimension of words.
\end{abstract}

\section{MOTS-CLÉS/KEYWORDS}

tourisme, lexiculture, mots à CCP, écarts culturels, pragmatique

Dans un dictionnaire du tourisme bilingue français-espagnol/espagnol-français, les informations culturelles peuvent bénéficier d'un traitement de choix, car même si le couple signifiant-signifié n'est pas forcément un élément de culture, le tourisme est une activité très riche du point de vue culturel où lexique et culture sont très souvent indissociables. Comme auteures ${ }^{1} \mathrm{~d}$ ' un dictionnaire du tourisme, il nous ${ }^{2}$ a paru intéressant et nécessaire, dans son élaboration, de faire ressortir l'aspect culturel de certaines unités lexicales, pour contribuer de la sorte à la formation socioculturelle de ses futurs utilisateurs.

Avant d'entrer dans le vif du sujet, des questions s'imposent à propos de cette tentative: pourquoi un dictionnaire et non pas un glossaire ? Pour qui est-il conçu ? Quel type de culture y est visé ? Quels mots ont une connotation spéciale ? Comment mettre en évidence les notions réellement partagées par les deux langues voisines et celles qui sont liées à la culture d'une des langues? Telles sont les questions auxquelles je tenterai de répondre dans les propos qui suivent, avant de montrer quelques exemples où langue et culture forment tout un binôme.

La présente recherche est née de deux observations : confectionner des listes de termes in abstracto ne nous attirait pas, d'abord parce qu'un simple index alphabétique de mots comprend rarement une composante culturelle, et d'autre part parce que de nombreux glossaires unilingues et bilingues existent depuis longtemps déjà dans le domaine du tourisme. Il fallait donc concevoir l'élaboration d'un dictionnaire que je qualifierais d' interstitiel ${ }^{3}$ pour deux raisons : en premier lieu, ce genre d'outil fait encore défaut à ce jour dans le secteur spécialisé du tourisme, en ce qui concerne les langues signalées supra. Il est donc nécessaire de combler ce vide (interstice) lexicographique; la deuxième raison est liée aux besoins particuliers des consultants potentiels en compétence culturelle, étant donné la large place qu' accorde le monde du tourisme à cette dimension. Alors pourquoi ne pas essayer de contribuer, ne serait-ce que modestement, à un accès à la culture dans cette entreprise dictionnairique ? En effet, un paramètre important dans la didactique des langues est de tenir compte des besoins réceptifs et productifs des usagers. Même si la 
centration sur l'apprenant n'est pas un concept nouveau, l'approche communicative est le premier courant méthodologique qui en a fait un pivot épistémologique. Ainsi, Un niveau seuil faisait jaillir la notion de besoin en dressant et croisant les publics et les domaines sociaux d'activité langagière pour conditionner des parcours différenciés. Dernièrement, Un Cadre européen commun de référence pour les langues qui préconise une perspective actionnelle, distingue également plusieurs sortes de domaines : personnel, public, professionnel et éducationnel. Ce Cadre indique également que certains domaines se chevauchent. On s'efforce donc dans les dernières décennies de proposer des formations correspondant à des besoins professionnels. Le français et l'espagnol du tourisme doivent concerner des destinataires professionnels, mais ceux-ci se croisent, somme toute, avec le monde éducationnel. Nous devions donc, dès le début, planifier un outil qui répondrait aux attentes des spécialistes du tourisme, des traducteurs, mais aussi des sujets d'un système éducatif varié, axé sur le tourisme.

Si une approche culturelle est toujours nécessaire, elle l'est davantage dans ce terrain spécifique, particulièrement porteur d'éléments culturels. Cependant, dans ce travail lexicographique, notre objectif n'est pas de former des consultants éclairés en connaissances pseudo-érudites; si parmi les champs lexicaux que le Dictionnaire du Tourisme (DT) comprend, il y en a un dénommé « culture », souvent associé à l'art, celui-ci ne présente pas généralement de difficulté au niveau des équivalences culturelles dans les deux langues; dans d'autres secteurs comme « général », « restauration », « spectacles », « logement », « jeu », « voyage », par exemple, notre souci majeur est de mobiliser, à travers les articles du DT un autre genre de culture, plus opaque pour les non natifs, et qui mérite que l'on s'y attarde ; c'est la culture-action, courante, expérentielle, partagée par la plupart, selon les néologismes créés par Galisson. Ce dernier définit ainsi cette culture réhabilitée et revisitée :

« Je définis la culture partagée ${ }^{4}$ comme une culture quotidienne transversale, une sorte de niveau-seuil comportemental du plus grand nombre, qui permet à l'immense majorité des natifs de se sentir des individus à part entière, et d'être reconnus comme tels par tous ceux qui se réclament de la même identité collective. L'identité collective étant le produit d'une langue et d'une culture partagées, donc d'un minimum de connaissances communes permettant à tous les membres d'une collectivité d'entretenir entre eux certaines relations de connivence, quels que soient leur niveau de scolarisation, leur appartenance socio-professionnelle, leur âge, etc.

La culture partagée est donc à la fois :

-un signe de reconnaissance tacite entre individus se réclamant de la même identité collective ; -un facteur de convivialité pour se comprendre à demi-mot[...] » (Galisson 1989 : 114).

Cependant, un problème surgit en ce qui concerne la culture courante en tant que produit de l'identité collective : comment un dictionnaire dit de spécialité, i.e. visant en principe un lexique spécialisé, utilisé dans notre cas par des spécialistes du tourisme, peut-il évoquer de la culture partagée par tous les membres d'une même collectivité linguistique ? Soulever la problématique des langues spécialisées mériterait une longue étude qui ne sera pas faite ici, mais je répondrai tout de même succinctement à cette question en reprenant l'avis de plusieurs auteurs, parmi lesquels Condamines et Rebeyrolles (1997 : 176), selon lesquels « une langue spécialisée ne constitue pas une langue à part entière parce qu'elle ne constitue en aucune façon un système autonome ». Dans le français du tourisme, cette affirmation acquiert encore plus de force, car si d'une part son lexique comprend un certain nombre d'éléments qui tiennent à la spécificité d'un contexte d'utilisation professionnelle, une grande partie de ce lexique n'appartient cependant pas à un domaine précis, mais à la langue générale ; les discours du tourisme sont alors plutôt que des discours spécialisés, des discours para-spécialisés, ayant fait l'objet d'une banalisation, et ils font donc partie pour la plupart de la culture expérentielle partagée par la communauté linguistique de la langue en question.

Le terme « culture » envisagé de la sorte s'associe à la forme « lexique » pour composer un autre néologisme cher à Galisson : la " lexiculture ${ }^{5}$ ». Il s'agit là d'une pratique lexicaliste conçue pour intégrer plus intimement l'enseignement de la culture à l'enseignement de la langue.

Toutefois, cette tâche s'avère difficile quand on a affaire à certains termes dotés d'un grand poids 
connotatif, d'une valeur ajoutée à leur signifié. Galisson confère à ces mots une « charge culturelle partagée $(\mathbf{C C P}) »$, et il en parle dans les termes suivants :

«La lexiculture [....] rend compte de la consubstantialité du lexique et de la culture, et désigne la seule valeur ajoutée aux mots par l'usage, valeur dont les dictionnaires ne rendent pas compte, et que je nomme CCP. En tant que didacticien, la CCP m'intéresse parce qu'elle constitue un obstacle majeur à la communication dissymétrique (entre natifs et non-natifs). En effet, l'implicite culturel, le non (explicitement) dit posent aux étrangers des problèmes aussi récurrents que difficiles » (Galisson 1995 : 5).

Ces mots à CCP sont donc de puissants accumulateurs de culture. Dans un dictionnaire du tourisme, ils relèvent de l'anthropologie culturelle comme par exemple les termes où se trouvent évoqués fêtes, spectacles, mœurs, hébergement. Il est donc très intéressant et utile de tenter de rendre compte aux étrangers de ces mots à connotation singulière et qui relèvent des savoirs partagés par le plus grand nombre des natifs. De cette façon, cette parcelle de culture vécue naturellement par les autochtones pourrait être décrite pour les usagers de la langue cible. La démarche à suivre consiste à mettre en oeuvre la pragmatique lexiculturelle, encore un concept opératoire attribué à Galisson en 1996. Son but est clair : " faire accéder autrement, à une autre culture, par un autre lexique » (Galisson 1999 : 478). « À une autre culture » fait référence à la priorité accordée à la culture courante sur la culture savante ; " par un autre lexique » signale la dimension pragmatique des mots, c'est-à-dire le produit de la relation que les locuteurs établissent avec ces mots à travers l'usage qu'ils en font.

Mais contribuer à la lexiculture dans un dictionnaire bilingue requiert tout d'abord une méthode au niveau de la microstructure, c'est-à-dire la présentation matérielle des articles ; en effet, les informations culturelles doivent être données de façon organisée, appuyées par deux critères essentiels : la clarté et l'économie. Dans ce domaine, la chance nous sourit car le département d'anglais de l'Université d'Alicante possède déjà une grande expérience en ce qui concerne l'entreprise dictionnairique, puisqu'il compte toute une série de dictionnaires spécialisés anglais/espagnol, espagnol/anglais, comprenant entre autres un dictionnaire du tourisme ${ }^{6}$. Enrique Alcaraz, qui dirige cette série et en est son concepteur, nous encouragea dans cette voie et nous permit de nous inspirer de son modèle microstructurel.

Il y a évidemment des mots-vedette qui ne posent aucun problème au niveau du décodage (compréhension); les articles qui leur correspondent sont courts et aucune information supplémentaire n'y est pertinente. On réalise dans ces cas-là une simple «traduction isomorphe » puisque les termes en contact de la L1 (langue source) et de la L2 (langue cible) sont équivalents dans les deux langues.

Cependant, dans le traitement des termes particulièrement affectés par une charge culturelle partagée, nous aurons affaire à une «traduction hétéromorphe », du moment où nous nous trouvons face à un terme L2 dont la relation n'est pas bijective avec celle du terme L1 ou si, même en remplissant la condition antérieure, il contient un sème nettement différent de celui de son homologue en L1. À cette occasion, il convient de construire un ensemble explicatif susceptible de regrouper, organiser et structurer les informations sémantiques présentes. Dans notre dictionnaire, les articles peuvent comporter une ou plusieurs des catégories suivantes :

- une traduction ou/et, selon les cas :

- une définition ;

- une glose culturelle, consistant en une explication succincte, s'il y a lieu ;

- un exemple, généralement une phrase-exemple, qui contextualise l'unité lexicale à élucider ou illustrer ; il est écrit en italique et il est précédé du symbole « $\div$ »;

- un relevé des environnements potentiels du terme en question, représenté par des expressions le comprenant. La traduction de celles-ci est toujours donnée entre deux tirets ; - des renvois synonymiques et analogiques en fin d'article, écrits en italique et précédés du symbole « V ». 
Ce sont les «traductions hétéromorphes » qui requièrent les articles les plus complets en catégories ; en effet, lorsque la traduction renvoie, au niveau pragmatique, à une réalité différente, on doit faciliter le maximum d'information au consultant afin de développer son savoir faire interprétatif en culture expérentielle.

À propos de la construction physique d'un article de dictionnaire, il existe certes nombre de règles et de principes à suivre, surtout en ce qui concerne la définition. Toutefois, rappelons qu'une terminologie qui tiendrait compte uniquement des principes prescriptifs n'assure en rien la garantie d'un article : « Nous ne gagnerons pas le paradis terminologique par l'achat d'indulgences typographiques » (Blanchon 1997 : 171). La clé du succès, selon cette auteure, est surtout fonction de la cible du dictionnaire, dans la mesure où l'on tient compte du public auquel il s'adresse. Étant donné les difficultés potentielles des futurs récepteurs au niveau de la compétence culturelle en langue 2, nous essaierons de les sensibiliser aux écarts culturels des deux langues en contact. Il y a en fait « écart culturel» lorsque le mot-vedette n'a pas d'équivalent dans la langue-culture cible, ou quand le terme a trait, au niveau pragmatique, à une réalité différente. Dans ce travail, nous distinguerons six cas de divergence que nous illustrerons au moyen d'exemples d'articles concernant d'abord des mots à $\mathrm{CCP}$, puis des cas divers de polysémie, de paronymes, de faux-amis et de glissements sémantiques. Nous les aborderons d'abord dans la perspective de l'approche intra-culturelle, puis inter-culturelle. Au lieu de joindre cet échantillonnage en annexe, je préfère le présenter à la suite, dans le corps de l'article, afin de commenter les résultats mis en évidence dans chacun des six points établis.

\section{Les écarts culturels : approche intra-culturelle (I)}

On ne prend en compte ici que la seule culture-cible.

\section{Termes-entrée sans équivalent dans la langue-culture d'accueil}

Les formes lexicographiques porteuses d'information culturelle peuvent n'appartenir qu'à un seul univers, comme les fêtes typiques d'un pays ou d'une région, la gastronomie, les loisirs ; ces unités lexicales n'ont pas souvent de correspondance dans l'autre langue et sont considérées intraduisibles, à moins que la langue cible n'ait recours à un emprunt.

beaujolais (REST vino francés del sur de la región de Borgoña; los más famosos son los jóvenes, afrutados y con una graduación de entre 11-12 grados; se toman el mismo año de su elaboración, y según la tradición, el beaujolais nouveau, llamado coloquialmente beaujo, se pone a la venta el tercer jueves de noviembre, constituyendo un gran acontecimiento en toda Francia $\div$ Le beaujolais nouveau est arrivé, allons le fêter!; V. vin, alcool; Bordeaux). Extrait 1 fr/esp ${ }^{7}$

cave $f^{8}$ (ESPECT cabaret situado en un sótano que se asemeja a una cueva; son célebres en París $\div$ Juliette Gréco est devenue célèbre en chantant dans les caves de Saint-Germain-des-Prés; V. caveau, cabaret, spectacle). Extrait 2 fr/esp

chocolatería $f$ (REST établissement où l'on sert la boisson de chocolat pour être consommée sur place; en Espagne, la coutume est de s'y rendre de bon matin, après une sortie nocturne, particulièrement après le «réveillon», pour y prendre un petit déjeuner avec des churros; certaines fabriques de chocolat ont leur propre « chocolatería » comme la marque Valor à Alicante et sa province; V. churros; marcha).

Extrait 3 esp/fr

marcha $f$ fam (GEN/SPECT sortie nocturne entre amis pour faire la fête jusqu'au petit matin; il s'agit d'un néologisme; on l'emploie dans des expressions telles que salir de marcha -sortir en boîte/faire la bringue- $\div$ Esta noche me voy de marcha con mis amigos; V. salida, baile; bebida, alcohol).

Extrait 4 esp/fr

\section{Mots à signifié commun, mais à $\mathrm{CCP}$ propre}

Un autre écart se situe dans les connotations liées à certains termes, car des mots semblables peuvent manifester des divergences d'emploi, à leur tour révélatrices de coutumes, représentations et 
comportements propres à une société déterminée. Ces particularités culturelles, implicites chez le natif, doivent être transmises au non natif dans le DT :

crêpe $f$ (REST crêpe; este galicismo alude a una oblea muy fina elaborada con una pasta hecha de harina, huevos y leche y cocida en una sartén de bordes bajitos; se puede utilizar en pastelería con azúcar, como la crêpe Suzette; en la festividad de la Chandeleur -candelaria-, el 2 de febrero, la tradición en todos los hogares de Francia es de hacer crêpes, haciéndolos saltar en alto, para darles la vuelta en la sartén; aparte de los crêpes dulces, existen también crêpes con preparaciones saladas $\div$ Cuando voy a Bretaña, me encanta comer crêpes, porque es una de las especialidades culinarias de esa región francesa; V. Chandeleur, galette).

Extrait 5 frlesp

muguet (PAISAJE muguete - lirio de los valles-; en Francia esta flor está unida a la festividad del primero de mayo; según la tradición, se regala ese día un ramillete de estas campanillas blancas, porque se cree que trae buena suerte; se utiliza en expresiones como brin de muguet -ramillete de muguete- $\div$ J'ai offert du muguet porte-bonheur à ma mère pour le premier mai).

Extrait 6 fr/esp

Le « jamón serrano », mot-étalon de la gastronomie espagnole

En ce qui concerne les extraits espagnols correspondants à ce point, $\mathrm{j}$ 'ai porté mon choix autour du terme « jambon », car le jambon cru peut être considéré en Espagne comme le produit ambassadeur de la gastronomie espagnole. D'ailleurs, il est très ancré dans les mœurs, étant même considéré comme le roi des « tapas », et on peut observer très couramment des pièces entières de jambon pendues dans les bars, charcuteries, boucheries. Il s'agit donc d'un mot hautement symbolique, à grande résonance culturelle, que je qualifierais pour cette raison de " mot-étalon » de la gastronomie espagnole. Le DT tient donc compte de ses variétés et catégories qui figureront sous la même entrée « jambon », mais que dans ce travail, j'ai préféré disposer à part :

jamón serrano (REST jambon cru de montagne; il existe, en Espagne différentes catégories de jambon cru qui varient en fonction de la race du porc et de l'alimentation à laquelle il est soumis; V. cuchillo jamonero, tabla de jamón). Extrait 7 esp/fr

jamón de bellota (REST jambon cru de la plus haute qualité qui procède d'un porc ibérico; le mot bellota signifie «gland » et indique que le porc n'a été nourri qu'avec des glands et des pâturages ; pour mieux apprécier sa saveur particulière, ce genre de jambon doit être coupé au couteau en tranches très fines; il y a même des professionnels spécialistes dans l'art de couper le jambon; V. jamón ibérico, jamón de recebo, jamón de bodega, jamón de pata negra).

Extrait 8 esp/fr

jamón de bodega (REST jambon cru de catégorie moyenne qui ne procède pas d'un porc ibérico; V. jamón serrano). Extrait 9 esp/fr

jamón de Jabugo (REST jambon de Jabugo; jambon élaboré à Jabugo, ville d'Andalousie, de la province de Huelva ; c'est le meilleur jambon cru d'Espagne; le label de qualité apposé sur un jambon de bellota peut indiquer selon la catégorie $3 j, 4 j$, ou $5 j$; V. jamón de bellota, jamón de pata negra, jamón ibérico).

Extrait 10 esp/fr

jamón de pata negra (REST jambon cru de grande catégorie dont le sabot est de couleur noire; les jambons de pata negra les plus connus sont des jambons de bellota et procèdent de Jabugo (Huelva) et de Guijuelo (Salamanque) ; V. jamón de Jabugo).

Extrait 11 esp/fr

jamón de recebo (REST jambon cru qui procède d'un porc ibérico, soumis à une alimentation mixte consistant en glands et pâturages, mélangés à des aliments composés; il est donc de qualité inférieure au jambon de bellota; V . jamón ibérico).

Extrait 12 esp/fr 
jamón ibérico (REST jambon cru de grande catégorie dont le sabot est généralement de couleur noire; les jambons «Ibéricos » les plus connus procèdent de Jabugo (Huelva), de Guijuelo (Salamanque) et de la Dehesa de Extremadura; V. jamón de pata negra, jamón de Jabugo, jamón de bellota, jamón de recebo).

Extrait 13 esp/fr

\section{Les paronymes}

Les paronymes sont très souvent source de confusion pour un étranger, puisqu'il s'agit de mots aux formes très ressemblantes. Ce sont en quelque sorte des faux-amis particuliers, internes, parce qu'ils ont lieu dans la même langue, comme il en est pour les termes suivants :

romain (ARTE/CULTURA romano $\div$ La ville de Nîmes compte de nombreux monuments romains comme la Tour carrée).

Extrait 14 fr/esp

roman (ARTE/CULTURA románico $\div$ J'aime beaucoup visiter en Provence les cathédrales romanes d'Avignon, de Cavaillon et de Saint-Trophime d'Arles; V. gothique).

Extrait 15 fr/esp

roman (ARTE/CULTURA novela $f ; \div$ Les Misérables de Victor Hugo est le roman le plus populaire de la littérature française; V. livre, nouvelle).

Extrait 16 fr/esp

estanco (GEN bureau de tabac [France], tabagie [Canada] ; bien que cet établissement soit destiné prioritairement à la vente du tabac, on peut y trouver bonbons, cadeaux, timbres, paris mutuels ; en France on y achète en outre des journaux et des billets de loterie à gratter ; en Espagne, par contre, on ne vend la loterie nationale que dans des établissements spécialisés appelés administración de lotería, où l’on fait également les paris mutuels ; V. expendeduría de tabaco ; tabaco, cigarrillos, puro).

Extrait 17 esp/fr

estanque (PAYSAGE étang, bassin [dans les jardins] $\div$ En los jardines del Retiro en Madrid, hay un estanque donde se puede remar en barca; V. lago, laguna, pantano, charca, alberca).

Extrait 18 esp/fr

Les écarts culturels : approche inter-culturelle (II)

Il s'agit dans ce cas, d'après Galisson (1999: 481), d'une "mise en contact de la culture-cible et de la culture-source, à partir d'observations ciblées sur des espaces culturels jugés a priori équivalents, de manière à relever de part et d'autre l'analogue dans le différent, le différent dans l'analogue et ainsi prendre conscience de la parenté des matrices à l'œuvre dans les cultures en contact ».

\section{Mots à signifié commun, mais à $\mathrm{CCP}$ différent dans chaque langue}

À ce propos, l'article du DT correspondant à l'entrée française donnera la connotation liée aux cérémonies religieuses et sociales (baptême, communion, mariage), tandis que l'article de l'entrée espagnole évoquera les fêtes de Noël.

dragée $f$ (REST peladilla $f$; en Francia el vocablo dragée está ligado a la tradición de regalar a los invitados de un bautizo, comunión o boda, cucurruchos o cajas de peladillas con el nombre de los homenajeados; según la tradición, en los bautizos son rosas para niña y azules para niño; las "peladillas" francesas son siempre alargadas $\div$ Je vais commander des cornets de dragées pour le baptême de ma fille).

Extrait 19 fr/esp 
peladilla $f$ (REST dragée $f$; les « dragées » espagnoles sont généralement plus rondes que les françaises ; elles font partie des confiseries de Noël ; V. dulces, turrón, piñones, mazapán, polvorón, mantecado).

Extrait 20 esp/fr

\section{Cas de polysémie bilingue}

\section{a) Polysémie non superposable : un seul terme d'une langue pour des équivalents différents dans l'autre langue}

Toutes les langues connaissent des éléments lexicaux polysémiques se servant des mêmes signifiants pour désigner des signifiés différents ; un dictionnaire bilingue passe obligatoirement par la désambiguïsation polysémique. Passons donc à délimiter les différentes sortes de polysémie que nous tentons de résoudre dans le DT. Précisons que certains articles présentent ici des entrées séparées pour un même terme (notamment « arroser » pour le français, et « estación » pour l'espagnol). Il s'agit pourtant bel et bien de polysèmes et non d'homonymes, puisqu'ils possèdent un étymon commun ( «arrosare » du latin populaire dans un cas, « statio » du verbe « stare » s'arrêter-, dans l'autre cas) et une cohérence sémique'. Encore une fois, ces signifiés divers d'une même forme polysémique seront rassemblés dans le DT sous une entrée commune. Quant à « tranche » pour le français et « pelar » pour l'espagnol, ils présentent un phénomène sémantique de particularisation lexicale, car la langue de départ ne possède qu'un seul mot, contre plusieurs termes voisins dans la langue d'arrivée qui différencient les nuances d'un même sens concret.

arroser col (REST mojar fam, convidar, celebrar, invitar a beber algo para celebrar un feliz acontecimiento $\div$ On va arroser la bonne nouvelle avec du champagne; V. inviter, fêter).

Extrait 21 fr/esp

arroser (REST rociar $\div$ On arrose souvent le poulet avec du vin).

Extrait 22 fr/esp

tranche $f$ (REST tajada $f$ [de carne, melón, sandía], rebanada $f$ [de pan], loncha $f$ [de jamón], rodaja $f$ [salchichón], filete [poisson]; V. pavé, rondelle, rouelle).

Extrait 23 fr/esp

déjeuner (REST almorzar [comer a mediodía, sobre las 14/15 horas en España, sobre las 12/13 horas en Francia], desayunar -pour le premier repas de la journée, on emploie aussi à sa place petit-déjeuner- ; V. petit-déjeuner, goûter, dîner).

Extrait 24 fr/esp

dîner (REST cenar [Francia], almorzar en la comida de mediodía [Canadá y Bélgica]; V. souper). Extrait 25 fr/esp

almorzar (REST déjeuner [vers 14 heures], manger quelque chose de léger [vers $11 \mathrm{~h}$,$] .$

Extrait 26 esp/fr

pelar (REST éplucher [légumes], peler [fruits] ; V. mondar).

Extrait 27 esp/fr

estación $f$ (VOYAGE gare $f$; on l'emploie dans des expressions telles que estación de ferrocarril -gare SNCF-, estación de autobuses -gare routière-, estación intermodal-gare intermodale- ; V. tren, autocar).

Extrait 28 esp/fr

estación $f$ (VOYAGE station $f$ [métro]; on l'emploie dans des expressions telles que estación terminal -terminus [métro, autobus]- $\div$ La próxima estación es Atocha; V. metro).

Extrait 29 esp/fr 


\section{b) Polysémie espagnole partiellement superposable à une lexie commune dans les langues}

\section{L1 et L2}

Dans « bungalow » et « cafetería », il convient de mettre en relief la plurivocité du terme-vedette, alors que dans la langue cible, la même lexie est monosémique :

bungalow (LOGEM bungalow; le sens coïncide dans les deux langues quand les termes font allusion à une maison simple à structure légère servant de résidence de vacances, implantée le plus souvent dans des hôtels, des villages vacances ou campings, au coeur des régions les plus touristiques ; cependant, de nos jours, le terme espagnol correspond la plupart du temps à une construction solide, sorte de pavillons jumeaux à deux ou trois étages, avec garage et petit jardin qui sert fréquemment de résidence principale et non secondaire ; V. chalet).

Extrait 30 esp/fr

cafetería $f$ (REST café, brasserie $f$; en espagnol, une cafetería fait généralement allusion à un café spacieux, à la décoration moderne où le personnel est souvent féminin; il peut aussi désigner la « cafétéria » française, c'est-à-dire un établissement en libre-service se trouvant par exemple en ville ou dans un centre commercial, hôpital, entreprise, faculté; on emploie souvent en français l'abréviation «cafèt »; V. café, bar).

Extrait 31 esp/fr

\section{c) Polysémie en L1 partiellement superposable à un homographe/homophone commun en}

\section{L2}

Dans les extraits qui suivent, il s'agit de nouveau de polysémie, et non d'homonymie, pour les mêmes raisons exposées supra, à savoir étymologie commune (les deux entrées

« lustre »proviennent du latin lustrare, à travers l'italien lustro) et liens sémantiques pour les motsentrée de chacune des deux langues en regard ; constatons que dans le cas de « sépia », la

polysémie s'explique par un glissement de sens de type métonymique, puisque la couleur sépia est obtenue à partir du liquide de la seiche.

lustre (GEN araña $f$-lámpara de techo- $\div$ Le lustre de cristal de ce salon est merveilleux; V. lumière, suspension). Extrait 32 fr/esp

lustre (GEN lustre, brillo $\div$ Je vais donner du lustre à ce cendrier en cuivre ; V. brillant, vernis).

sepia $f$ (ART sépia $f$-matière colorante d'un brun foncé extraite du liquide de la seiche- $\div$ Conservo unas viejas fotos de mis abuelos de color sepia; V. color, pintura).

Extrait 34 esp/fr

sepia $f$ (REST seiche $f$; en espagnol, on dénomme ce mollusque jibia dans certaines régions $\div$ Me encanta la sepia a la plancha; V. jibia; pescado, calamar, pulpo).

Extrait 35 esp/fr

\section{Faux-amis}

J'ai préféré disposer à part les faux-amis qui se produisent entre deux langues différentes (les paronymes qui sont des faux-amis « internes » sont traités au point 3) ; Par ailleurs ceux que j'inclus ici sont considérés comme des faux-amis « complets », par opposition aux « partiels » qui correspondent aux termes polyréférentiels (ces derniers ont déjà été analysés au point 4).

mallette $f$ (VIAJE neceser -caja o estuche rígido que sirve para guardar lo necesario para el aseo personal- , cartable d'écolier [Belgique]; il ne faut pas confondre mallette avec le terme español « maleta »dont l'équivalent français est valise; $\mathrm{V}$. trousse de toilette).

Extrait 36 fr/esp 
bonbon (REST caramelo; se utiliza en expresiones como bonbon au lait -caramelo de leche-, bonbon à la réglisse caramelo de regaliz-, bonbon fourré -caramelo relleno-; on ne doit pas confondre bonbon avec le mot espagnol

«bombón » qui signifie chocolat; V. sucreries, friandises).

Extrait 37 fr/esp

vianda $f$ (REST nourriture $f$; au pluriel viandas fait allusion à « mets »; on ne doit pas confondre vianda avec le mot français « viande » qui signifie carne; V. comida).

Extrait 38 esp/fr

Potaje (REST espèce de ragoût composé principalement de féculents et de légumes ; on ne doit pas confondre potaje avec le terme français «potage » qui désigne une soupe $\div$ Voy a comer hoy un potaje de garbanzos; V. guiso, guisado, cocido).

Extrait 39 esp/fr

\section{Glissements sémantiques dans les emprunts}

Les emprunts représentent un autre cas particulier de faux-amis, qui peuvent être également source d'erreurs ; certains mots étrangers sont des calques qui ont subi un déplacement de sens dans la langue réceptrice ; quelquefois l'utilisation d'un terme effectuée dans une des langues est très restreinte et correspond à un usage technique : ainsi, l'unité lexicale « petit menaje » n'existe que dans le vocabulaire spécialisé de l’hôtellerie espagnole et ne correspond à aucune des acceptions du terme français « ménage ».

\section{a) Gallicisme ayant un sens différent en espagnol}

petit menaje (REST table à condiments $f$-ensemble de sauces, épices, salière, huilier, moutarde, etc., utilisé pour le service au client- ; V. salsa, especie, salero, vinagrera, convoy, mostaza).

Extrait 40 esp/fr

\section{b) Hispanismes ayant un sens différent en français}

gamba $f$ (REST carabinero; on emploie gamba en français pour désigner les grosses crevettes espagnoles ; V. crevette, langoustine).

Extrait 41 fr/esp

\section{Conclusion}

L'intérêt de ce bref parcours n'est pas de présenter un état des lieux, mais d'une part de mobiliser et actualiser la culture dans et par les mots, et d'autre part de mettre en contact et en contraste certaines réalités des deux langues en présence pour attirer l'attention du lecteur sur les symétries trompeuses. En effet, le français et l'espagnol considérés comme des « langues voisines » ou «cousines » ont certes des systèmes proches, et cette proximité peut être réellement un facteur favorable dans l'apprentissage, mais elle peut être aussi source d'interférences et rendre d'autre part plus difficile la découverte de l'altérité.

De plus, en familiarisant le consultant du DT à la culture partagée, il en résulte un avantage supplémentaire : on l'amène de la sorte à relativiser sa culture maternelle, en lui permettant de s'interroger sur les savoirs constitutifs de l'autre culture, et par voie de retour, on suscite chez le lecteur une réflexion sur sa propre culture. Prendre conscience de la pluralité, sans dévalorisation, ni survalorisation, suppose un enrichissement des points de vue. Un dictionnaire du tourisme peut devenir ainsi un lieu d'observation de la culture, et comme préconise Charaudeau, « un médiateur entre la culture des apprenants et celle de la langue enseignée ». Notre ambition n'est autre que de fournir un outil de réflexion, d'action et non seulement de traduction, de façon à aider le lecteur à construire lui-même ses propres informations ou connaissances.

Cependant, un dictionnaire conçu de la sorte, comme un dictionnaire d'apprentissage, s'expose à des dangers : il n'échappe pas à l'arbitraire, car témoignant quelquefois d'une observation empirique que la dictionnairique classique ne recueille pas toujours, les informations 
culturelles fournies répondent à une certaine subjectivité, relevant d'expériences de la vie sociale, perçues par les auteures d'une façon intuitive.

Un autre facteur arbitraire, au niveau de la macrostructure, concerne le choix des entrées culturelles, qui peut être questionné à juste titre : il manque sans doute nombre de lemmes culturels importants, et le DT s'avère sans doute un ouvrage fragmentaliste et non pas exhaustiviste, mais on ne peut tout embrasser dans un dictionnaire de ce genre. D'autre part, il faut reconnaitre en outre, une limitation au DT : il ne cerne que le français utilisé dans l'Hexagone, sauf exceptions, ce qui est bien dommage dans le cadre du colloque de Montréal.

Nous sommes donc conscientes de la difficulté de cette entreprise dictionnairique, et nous nous contentons de présenter de la langue culture et non de la culture, car ce travail n'est qu'une simple contribution à la pragmatique lexiculturelle d'un dictionnaire bilingue du tourisme.

\section{NOTES}

1. Ce dictionnaire du tourisme, dont les autres auteures sont Mercedes Eurrutia et Montserrat Planelles, n'est pas encore sorti au moment de la rédaction de cet article.

2. Le pronom « nous » employé au long de ce travail correspond au pluriel de majesté, et représente donc les trois auteures ; pour évoquer une démarche personnelle, je recourrai au pronom « je ».

3. La catégorie des dictionnaires interstitiels est citée par Galisson (cf. « Une Dictionnairique à géométrie variable au service de la lexiculture » (Galisson 1997 : 60).

4. Cette expression figure en caractères gras dans l'article cité de Galisson.

5. Galisson a créé le concept de lexiculture. Il désigne ainsi, en 1987, la culture véhiculée par les mots à CCP.

6. Cf. Alcaraz (2000).

7. Dans cet article, pour mieux distinguer visuellement la partie espagnole de la partie française, les extraits françaisespagnol sont placés à droite, tandis que ceux concernant les extraits espagnol-français sont à gauche.

8. Par souci d'économie, nous ne notons que le genre féminin $f$.

9. À propos des critères étymologiques et sémantiques pour caractériser la polysémie, cf. p. ex. Martinet 1974, Lyons 1990 et Picoche 1984.

\section{RÉFÉRENCES}

Alcaraz Varó, E. et al. (2000) : Diccionario de términos de turismo y ocio Inglés-Español/Spanish-English, Barcelona, Ariel.

Blanchon, I. (1997) : « Point de vue sur la définition », Meta 42-1, p. 171.

Condamines, A. et J. Rebeyrolle (1997) : « Point de vue en langue spécialisée », Meta 42-1, p. 176.

Coste, D., J. Courtillon, V. Ferenczi, M. Martin-Baltar, E. Papo et E. Roulet (1979) : Un niveau seuil pour des contextes scolaires, Paris, Hatier.

CONSEIL DE L'EUROPE, DIVISION DES LANGUES VIVANTES (2000) : Un Cadre européen de référence pour les langues : apprendre, enseigner, évaluer, Strasbourg, Conseil de L'Europe.

GALISSON, R. (1987) : «Accéder à la culture partagée par l'entremise des mots à CCP », ÉLA 67.

GALISSON, R. (1989) : « La culture partagée: une monnaie d'échange interculturelle », Lexiques, coll. « F », p. 114. GALISSON, R. (1995) : «Où il est question de lexiculture, de Cheval de Troie et d'impressionnisme... », ÉLA 97, p. 5 .

GALISSON, R. (1999) : «La pragmatique lexiculturelle pour accéder autrement, à une autre culture, par un autre lexique », ÉLA 116, p. 478.

LyONS, J. (1990) : Sémantique linguistique, Larousse, Langue et langage.

MARTinet, A. (1974) : «Homonymes et polysèmes », La linguistique 10, p. 37-45.

PICOCHE, J. (1984) : «Recherche de la cohérence des divers emplois du signe linguistique (fondamentale chez Guillaume) à travers la notion de signifié de puissance », Cahiers de lexicologie 44-1. 